\title{
Mobile Manipulation using NASA's Robonaut
}

\author{
Robert O. Ambrose, Robert T. Savely, S. Michael \\ Goza, Philip Strawser \\ NASA Johnson Space Center \\ Automation, Robotics and Simulation Division \\ Houston, Texas 77058
}

\author{
Myron A. Diftler, Ivan Spain, Nicolaus Radford \\ Lockheed Martin \\ Automation and Robotics Department \\ Houston, Texas 77058
}

\begin{abstract}
The Johnson Space Center has developed a new mobile manipulation system with the combination of a Robonaut upper body mounted onto a Segway mobile base. The objective is 0 study a fluid and coordinated control of dexterous limbs on a mobile robot. The system has been demonstrated interacting with people, tools, and urban interfaces built for humans. Human interactions have included manually exchanging objects with humans, following people, and tracking people with hand

- held objects such as flashlights. Like other configurations of the Robonaut family, the upper body provides dexterity for using tools such as wire cutters, shovels, space flight gear, and handling nexible tethers and fabrics. The Segway base is a custom version called the Robotic Mobility Platform (RMP) built for DARPA, and provided to NASA for this collaborative effort. The RMP's active balance gives Robonaut a relatively small footprint for its height, allowing it to pass through doors and elevators built for humans, and use wheelchair accessible ramps and lifts. Lessons learned from this development will be presented to improve the design of future mobile manipulation systems, and the Segway base provides mobility to Robonaut for Earth based testing.
\end{abstract}

Keywords-Mobile, Manipulation, Robonaut, Segway, Space

\section{INTRODUCTION}

The system was developed as a NASA/DARPA collaboration to investigate mobile manipulation systems. DARPA IPTO worked with Segway LLC to develop the lower body, and with NASA JSC to develop the upper body. JSC integrated the system in the summer of 2003 , and the system came on line August 25, 2003.

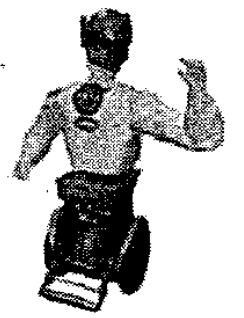

Figure 1. Photo of Robonaut on RMP

The primary development objective for the mobile Robonaut system was to identify control options for teleoperation and autonomous control of a dexterous, dual arm robot that is mobile. A secondary objective was to investigate this wheeled configuration of Robonaut for terrestrial service roles such as bomb disposal, plant maintenance, and security. Figure 1 shows the system as a combination of the Robonaut and RMP robots.

\section{A. Background on the Segway RMP}

The Segway ${ }^{\mathrm{TM}}$ Robotic Mobility Platform (RMP), as shown in Figure 2, is a derivative of the Segway ${ }^{\mathrm{TM}}$ Human Transporter (HT). The HT was designed by Segway LLC to be a two wheeled motorized vehicle for transportation. DARPA later commissioned Segway LLC to develop the RMP, which is a computer-controlled version of the HT capable of balancing large payloads. The only external inputs required for controlling the RMP are velocity and turn rate. When these values are set to zero, the RMP will hold position even when extemal forces are applied. One of these very special devices was delivered to NASA JSC in July 2003.

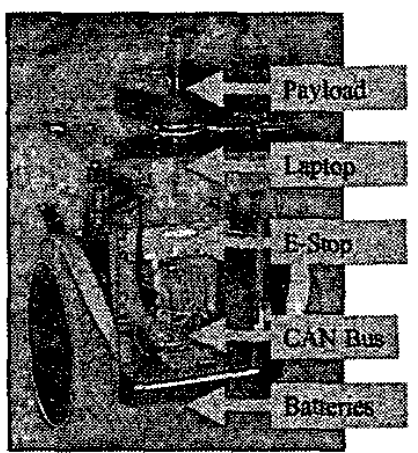

Figure 2. Photo of Segway RMP

\section{B. Background on Robonaut}

NASA has now completed two Robonaut prototypes. The first one, Robonaut A [1], was built as an evolving system over many years, as shown in Figure 3, with progression from arms and hands, to an integrated single limb system, to an upright, dual arm, upper torso. Robonaut $\mathrm{B}$ was completed in the Fall of 2002, and was built in a single production. These prototypes 
have been built following a philosophy that will enable future space flight of the design, by making careful design choices on materials.

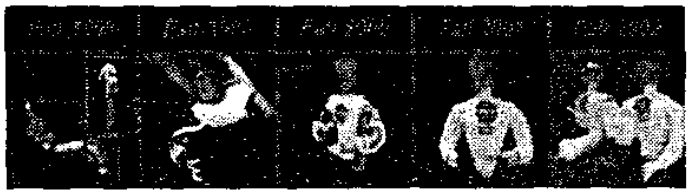

Figure 3. Robonaut Development History

To save cost, many components have been selected based on the availability of flight-qualified options, though the prototypes use the less expensive, non-flight versions. Other subsystems, like arm joints, have been tested in thermal vacuum chambers at JSC and found to work well across the extreme temperature ranges found in space.
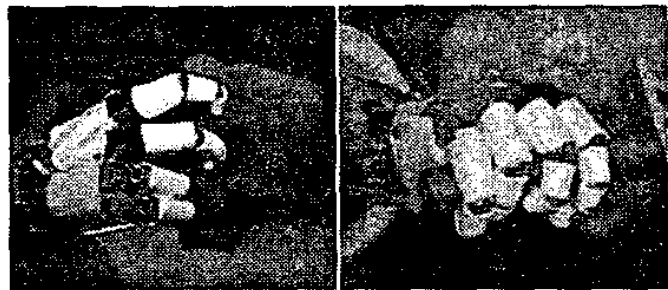

Figure 4. Robonaut Handling a Rock and Electrical Connector

One of the fundamental goals in designing Robonaut was to allow the robot to accommodate existing interfaces built for humans [2]. This reduces the need for a second set of tools for robot and human, and allows the robot to work on existing spacecraft. The Robonaut design has met the challenge of creating a general manipulation system, motivated by the requirements of existing spacecraft that were built for humans. Figure 4 shows Robonaut $A$ handling general objects that can be grasped by suited humans.

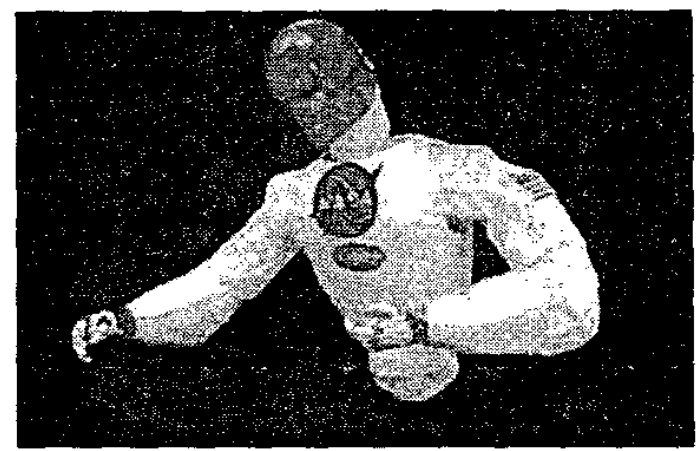

Figure 5. Robonaut A reaching with Arms and Waist

\section{Robonaut A Configuration}

Figure 3 shows the evolution of Robonaut $A$, the first Robonaut that was built at JSC. The final integration of Robonaut A resulted in a 44 Degree of Freedom (DOF) upper torso mounted on a 3 DOF waist. Since 2000, Unit A has been over $90 \%$ available, and used in numerous autonomy, human- robot interaction and telepresence studies [3]. The limited waist mobility of the system, though fixed on a pedestal, was found to greatly increase the workspace of the robot. Shown in Figure 5, the reachable and dexterous workspaces of the upper arms can be reoriented around the pedestal, expanding the system's sphere of influence.

\section{Robonaut $B$ Configuration}

During EVA, crewmembers often place both legs into a portable foot restraint connected to either a Remote Manipulator System (RMS) or a special socket on the spacecraft. This creates a path to react the loads generated during work. In its microgravity configuration, Robonaut B works on the same principle. Instead of a foot restraint to react loads, Robonaut B has a single seven DOF leg with the same interface to the spacecraft foot restraints as an end effector, Figure 6. Once anchored to a spacecraft, the multi-jointed leg provides a greater amount of body mobility than even a human crewmember. Additionally, Robonaut $\mathrm{B}$ is designed to have a grapple fixture on its back, allowing it to be maneuvered by an RMS. When utilized in this manner, the leg is a third arm.
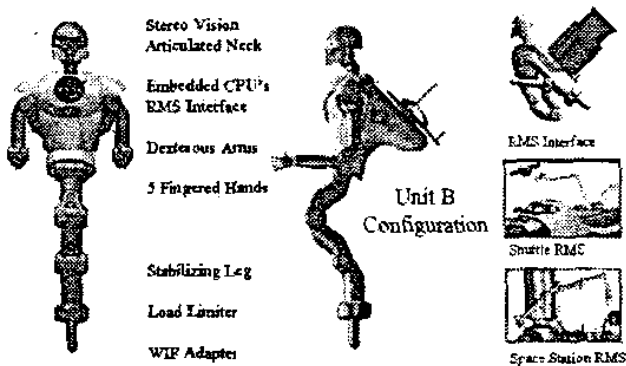

Figure 6. Robonaut B Anatomy and Applications for working in Space

Robonaut $\mathrm{B}$ has this added zero-G stabilizing leg for use on the exterior of spacecraft, a fully integrated avionics suite in its torso, an interface on its back for the larger Space Shuttle and Space Station arms (RMS and SSRMS, respectively) and added dexterity in its neck.
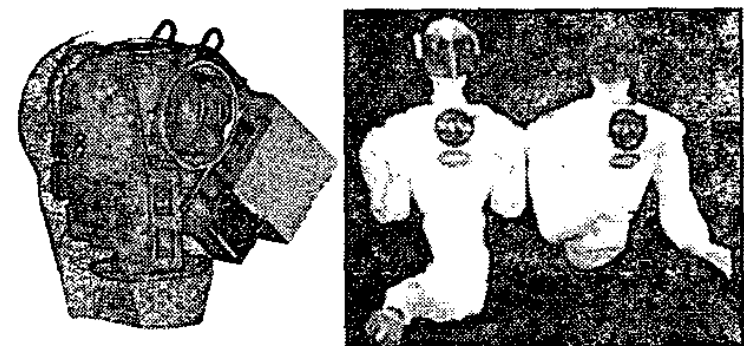

Figure 7. Robonaut B Body Packing, and Comparison to Robonaut A

Figure 7 illustrates that both Robonauts are similar in appearance. However, Robonaut B is not merely a copy of Robonaut A. The team had many new ideas on component technologies, and the challenge of making it portable drove it to new designs. In particular, Robonaut $B$ has fully integrated avionics, with the large rack behind Robonaut $A$ now 
miniaturized, and completely packaged in the robot's torso, as shown in Figure 7. A cart makes Robonaut B portable, allowing the team to move the robot out of the lab and transport it off-site. Due to the embedded avionics, the interface to the robot is now simplified to raw power and Ethernet; a dramatic change over Robonaut A's many hundreds of cables. The embedded avionics also includes a complete, 8 slot $\mathrm{cPCl}$ chassis that is unallocated, ensuring that the robot can grow with new autonomy requirements now being researched using Robonaut A.

\section{SYSTEM OVERVIEW}

Robonaut B's upper body was used to assemble this mobile configuration. Being portable and self contained, Robonaut $B$ still required a number of modifications for this integration. The main efforts were to develop an onboard battery power system, and to provide numerous wireless communication channels for data and video. During this work, safety was a key consideration, with a heavy and valuable robot being placed on a research platform. Another area of new work was in the integrated control of the mobility base and the dexterous upper body. Each of these challenges will be described.

\section{A. Onboard Power Control System}

The Mobile Power Control System (MPCS) is comprised of - a central power conditioner, which provides all relay logic and power control to the upper and lower body. Since safety is always of utmost concern, power control relay logic was implemented to keep high power systems from turning on without the presence of proper computer control.

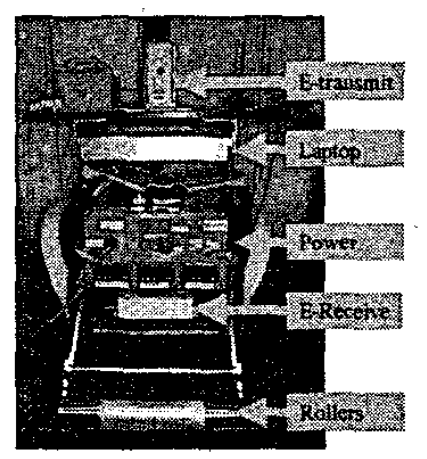

Figure 8. Photo of Robonaut's RMP Control Panel

The MPCS delivers several power buses to control all of the robot's systems. An isolated DC-to-DC switching regulator steps down $72 \mathrm{VDC}$, provided by two nickel metal hydride batteries, to 28 VDC for all the upper body computer and logic systems. These two batteries are the same type of batteries used on the HT and the RMP. There is also a separate motor power bus that contains 28VDC for all hand motor packs and brakes. A special feed through directly connects the batteries to all the high voltage motors. The SPCS itself has three current displays, which give readings of these three main power buses (28V Computer, $28 \mathrm{~V} \mathrm{Motor/Brakes,} \mathrm{HV} \mathrm{Motors)} \mathrm{at} \mathrm{all} \mathrm{times.}$ The current meters, current sensors, wireless video, and the wireless E-stop receiver box are all powered from $\mathrm{a}+/-15 \mathrm{~V}$ DC-to-DC switching regulator off the main battery bus.

\section{B. Onboard Wireless System}

The wireless system transfers three types of information: robotic command and control, video, and the emergency stop control. Robotic command and control is handled over a wireless Ethernet that consists of two distinct channels. One channel is used for controlling the upper body and the other is for Robonaut's lower body, the RMP. In the upper body, the PowerPC processor card has an Ethernet port which is hard wired to a wireless Ethernet access point. This access point, in turn, communicates with the remote workstation's wireless network router. In the RMP, a laptop computer communicates directly to the wireless network router through a wireless PCMCIA Ethernet card.

The wireless video system also consists of two channels. Each video channel is tied to one of the robot's cameras (Robonaut's eyes) inside the head. Each camera transmits video from a unique transmitter to a common patch antenna video receiver. The patch antenna network is configured to eliminate signal multi-pathing issues as well as to enhance signal clarity. The video signals are sent from the receiver to the headset of the teleoperator.

The wireless emergency stop (e-stop) control is transmitted on two separate channels. One channel is used for the upper body (the arms, waist, hands, and head) and the other channel is used for the lower body (the RMP). The wireless e-stop is broken into two channels to allow the robot and its mobile base to be shut down independently. Each channel has an associated hand-held box operated by a human safety supervisor. The hand-held e-stop boxes will shut down the associated subsystem for one of two reasons: 1) the robot goes out of range of the hand-held e-stop, 2) the human safety supervisor presses the button on the hand-help e-stop box.

\section{Safety}

The mass of the Robonaut upper body is $105 \mathrm{~kg}$, and the mass of the lower body is $45 \mathrm{~kg}$, as built and weighed prior to final integration. The top speed of the robot is $4 \mathrm{~m} / \mathrm{s}$, which can be limited to lower values through software controls. The robot will be operated in tight confines, and near people. The upper body is a particularly valuable piece of equipment. The objective of operating the robot will be to intentionally make contact with the environment for mobile manipulation, not avoid contact like in most navigation problems. All these factors require the robot to be treated with care. Therefore a detailed safety analysis was done prior to design. A careful test plan was also developed to test out each element in the safety system.

The RMP was delivered with a leash style safety cord, where a person can pull the cord, breaking a contact, and causing the RMP to shut off. The robot can also be commanded to shut off over the CAN Buss interface from the onboard laptop. Both of these have problems. The leash requires a person to be relatively close to the robot, and can become caught in or under the wheels of the robot. The commanded power down is dependent on the health of the 
CAN Bus, the laptop, and any communication between the laptop and off board processes and supervisors.

An independent safety system was developed and installed on the robot. The wireless emergency stop (E-Stop) system previously described was inserted into both the RMP power control, and the power system for the upper body. These transmitters have sufficient range to keep people out of harms way, are fail safe, and in series with the commanded stop options already present in the RMP and Robonaut subsystems.

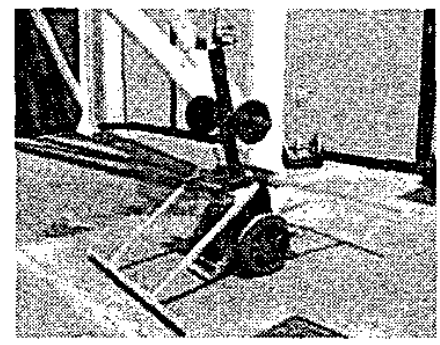

Figure 9. Testing with Weights and Safety Bars

Because the RMP is an active balance system, it needs power to remain upright and safe. Shutting off the RMP results in the robot falling to the ground. A robot of this mass falling in this way, potentially on concrete, would be devastating. To remove this risk, rollers were built to serve as landing gears. Shown in Figure 8, these can be adjusted to provide a varying degree of pitch range for the base. Robonaut's RMP is currently operated with a $t /-12$ degree range. When power is removed, the robot tilts only a very short distance, and lands safely on this roller.

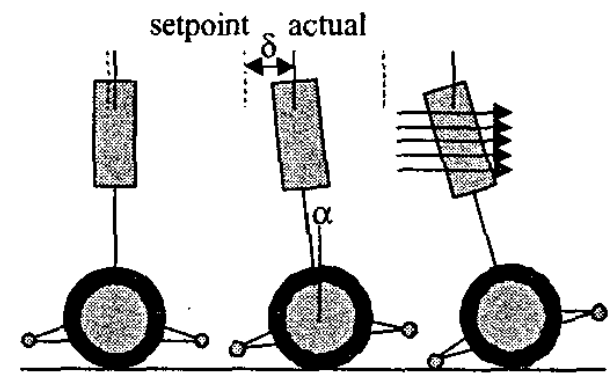

Figure 10. RMP Balance Conditions (Standing, Driving, Runaway)

The rollers provided another form of safety, by allowing the robot to be worked on in a nominally upright position, while inactive. But, the rollers were found to induce an instability in the control law for balance. When the robot leaned to a desired angle beyond which the current rollers' settings would allow, the robot would accelerate proportional to the controller's proportional gain. This was a significant danger, and verified in early testing with the RMP unloaded and then with added weights. Figure 10 shows three cases of balancing state. In the first state, the setpoint position is near the actual position, and the system balances within a nominal steady state error. In the second case, the setpoint is to the left of the actual position. The robot leans to the left, to provide thrust in that direction, and begins a leftward pursuit of the setpoint. An interesting observation is that to provide leftward thrust, the robot base drives to the right, inducing the leftward lean, then accelerates left. The last case has the setpoint to the left of the actual, but the roller in contact with ground. In this case, the robot is unable to lean to the left, but goes to full throttle in its attempt to do so, accelerating rightward.

To protect the system from this case, the data coming from the RMP gyroscopes was used to detect the pitch angle. Since the rollers are adjustable, the angle of the roller can be known, and a software limit angle can be defined prior to (less than) that angle. For current operations, the rollers are set to an alpha angle of 12 degrees, and the software angle limits are set to 10 degrees. If the software limit is ever exceeded, the RMP gently shuts down. This was tested extensively, prior to Robonaut B being mounted on the RMP.

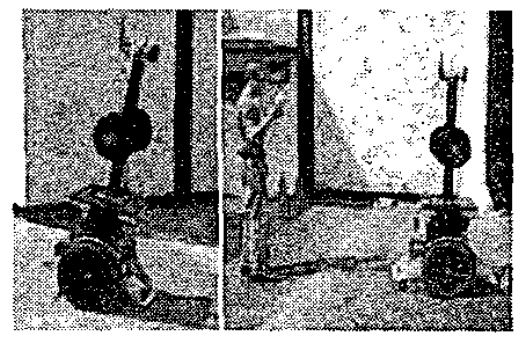

Figure 11. Dynamic Testing with Weights at Worst Case Height

The balance algorithm has the option of three gain settings that vary assumptions about payload mass and teight. The combined system was weighed, and the center of gravity (CG) was measured experimentally. Given the limited choices, the large mass, and high CG setting was selected. Early testing of the RMP was performed with a set of weights that were positioned to emulate the Robonaut mass and CG. Figure 11 shows the system with the weights positioned higher than the case where Robonaut's arms are raised. In this case, the control algorithm was experimentally found to have a $0.5-0.6$ $\mathrm{Hz}$ natural frequency. The leftright roll mode is uncontrollable, and is due to the mass and compliance of the tires. This mode was found to be $1.3-1.6 \mathrm{~Hz}$. These low modes were further suppressed by the addition of the outriggers shown in Figure 9. Their mass and inertia were found to induce lightly damped oscillations at $0.4 \mathrm{~Hz}$ in pitch. Another finding was that a point load mass, placed at a specific height, would cause instability if the system began to pitch about that point. Distributing the same mass, but with a larger moment of inertia about that pitch axis, would reduce the oscillation. Robonaut's mass is well distributed, and was found to work better than the weight testing.

\section{Coordinated Control for Mobile Manipulation}

Through collaboration with DARPA, the Robonaut team is now working with researchers at Vanderbilt, UMass, MIT, and USC to develop a mix of autonomy skills for dexterous handling of tools, with little or no supervision by humans [4]. The vast majority of this work has used Robonaut $A$ as the autonomy testbed. At the current time, the majority of operations have been conducted using Robonaut's telepresence control mode. 
Robonaut uses a mix of telepresence interfaces [5]. Most are chosen for comfort, ease of egress, and minimizes external hindrances with the physical environment. Therefore any sort of exoskeleton type hardware was immediately dismissed. The operator dons a myriad of virtual reality (VR) hardware to fully immerse and become Robonaut. The VR gear utilized on Robonaut consists of a helmet, gloves, body tracking and foot pedals. Everything but the foot pedals are visible in Figure 12. All of this equipment works in concert to give the operator the illusion of actually being the robot in a comfortable fashion. This also provides the added benefit of creating a very intuitive interface that is easy for novice operators to understand.

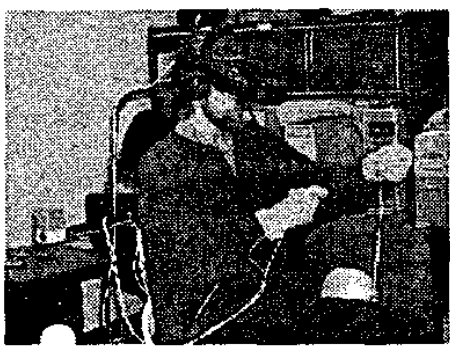

Figure 12. Robonaut Telepresence Gear

Robonaut has two pairs of cameras mounted in its head. By feeding the images to a VR helmet, the teleoperator perceives a stereo view of the world from the perspective of the robot. This view is key to the effectiveness of the teleoperator. "By using depth cues gleaned from stereo images and lighting, the operator develops a sense of the environment. In fact, the images are so compelling that operators have been known to jerk their feet back in response to falling objects. This is usually an amusing situation considering the operator is nowhere near the robot. This state of immersion is highly desirable when controlling the Robonaut. When immersion occurs, the operator no longer considers the robot a separate entity. It is now part of his/her body, which increases productivity.

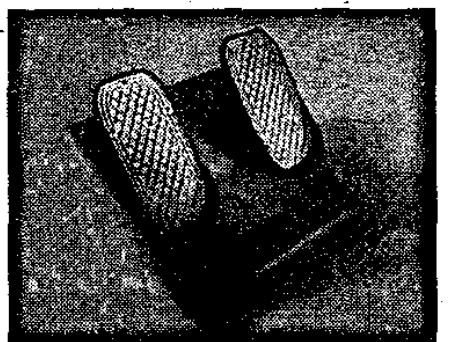

Figure 13. Foot Pedals used to Operate the RMP

Since the teleoperators hands and upper body are instrumented for control of $47 \mathrm{DOF}$, the only part left uninstrumented is the feet. The feet control the 2 DOF mobility of the RMP. CH Products ProPedals ${ }^{\mathrm{TM}}$, see Figure 13, flight simulator foot pedals are used to control the base of Robonaut. Initially a tank style foot pedal control was implemented. Tank steering is where the travel of the pedals is centered. Pressing both feet forward would cause forward motion. Both back would cause reverse movement. One forward, one back would cause turning. This method was poblematic based on the hardware. The foot pedals on a flight simulator are not centered in their travel. They are more like the pedals on a car or aircraft. The foot pedals are sprung in one direction. They also move fore and aft about a central pivot just like the rudder pedals on an airplane. Therefore, tank steering was physically impractical.

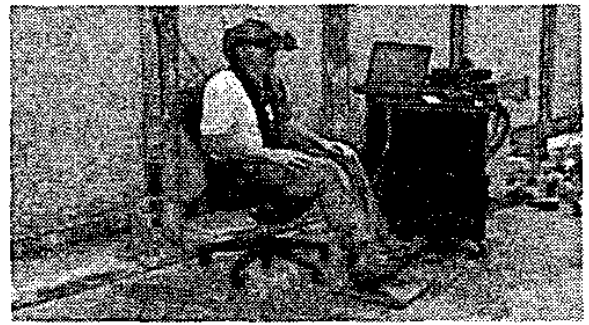

Figure 14. Teleoperator Controlling the Full Segwanut System

\section{WAIST DESIGN}

The tail of Robonaut Unit B, except for a single roll joint, was removed in preparation for mounting it on the RMP. This remaining joint provides a 1 DOF waist and allows Robonaut to pivot on top of its mobile platform, providing more flexibility to the teleoperator. Prior research [6] has shown that combining a small pair of dexterous arms on a waist is more effective than two large arms, rigidly mounted to the base. This exploration of a bifurcating structure has been explored on Robonaut A [7], and analyzed in simulation.

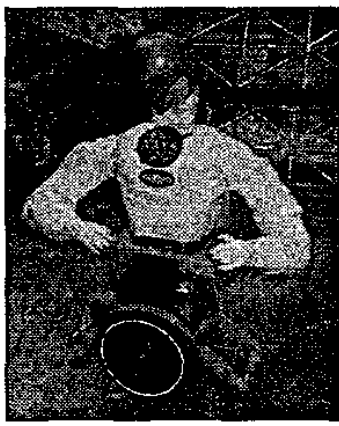

Figure 15. Waist Motion

Figure 15 shows the upper body rotated relative to the RMP, using this waist motion. An example of application would be to orient the body along a wall, as the lower body drives along the base of the wall. The fact that the neck can pan about a parallel axis allows three options for turning the head: tum with the RMP, turn with the waist, or turn with the neck. This redundancy is mapped to the RMP velocity vector, the center of the arm's dual workspace, and head gaze vector.

\section{IV, TASKS}

A series of Experiments have been performed with the mobile Robonaut system. The following series of photos demonstrate the range of capabilities that are present in this versatile machine. 


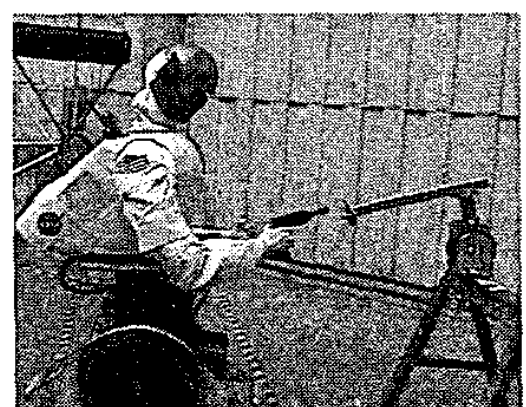

Figure 16. Tightening Lug Nut with Torque Tool

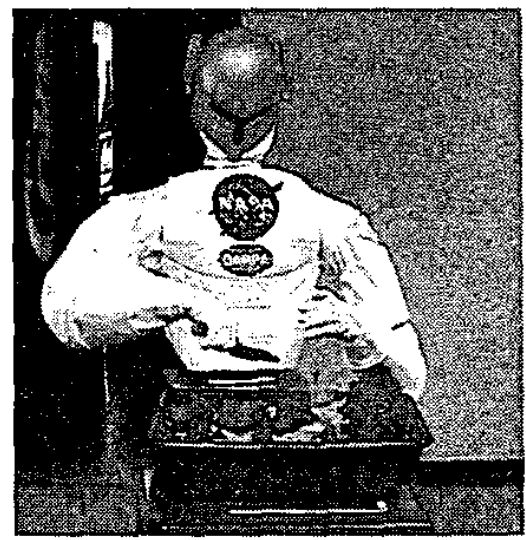

Figure 17. Digging with Hand Shovel

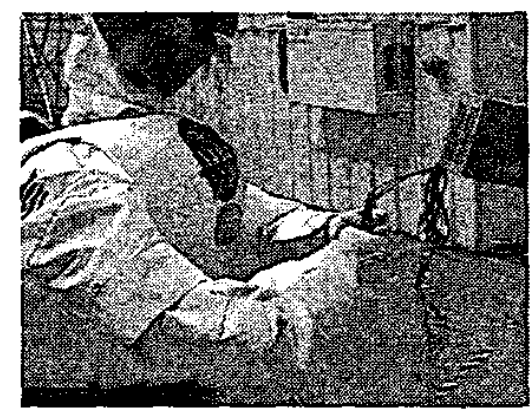

Figure 18. Cutting Wires

\section{CONCLUSIONS}

This new configuration of Robonaut has an unprecedented combination of mobility and dexterous manipulation. Its top speed is faster than any biped humanoid by a factor of 3 . lts dexterous limbs can manipulate tools, and carry objects. It can work safely with people, and negotiate tight confines. Future work includes adding the autonomous skills under development on the other Robonaut systems, and expanding the waist mobility to include other degrees of freedom.

One benefit of a mobile Robonaut is its ability to assume burdens and risks by venturing into hazardous environments. Through advanced telepresence and automation, an operator may apply their human problem solving abilities without coming into harms way. Robonaut is distinctively different from other robots placed into hazardous situations because it is designed to work in human environments with human tools.

\section{ACKNOWLEDGMENT}

This work was funded by Dr. Douglass Gage of DARPA's IPTO, under the MARS program and Mr. David Lavery of NASA Code S.

\section{REFERENCES}

[1] Ambrose, Robert O., Aldridge. H., Askew. R. S., Burridge. R.. Bluethman, W., Diftler. M.A., Lovchik, C.. Magnuder. D. and Rehnmark, F., "ROBONAUT: NASA's Space Humanoid", IEEE Intelligent Systems Joumal. August 2000

[2] Whitakker, R., Staritz, P. Ambrose, Robert O.. et. al., "EVA Robotics for Space Solar Power Stations", Journal of Aerospace Engineering of ASCE Special Issue on Space Solar Power.

[3] Ambrose, R.O., Culbert, C., and Rehnmark, F." An Experimental Investigation of Dexterous Robols working with EVA Hardware". AIAA Space 2001, Albuquerque Nm, August 2001.

[4] Diftler, M.. Culbert, C., Ambrose, R.. Platt. R.. Bluethmann, W., "Evolution of the NASA/DARPA Robonaut Control System", ICRA 2003, Taipei, Taiwan, May 2003.

[5] Diftler, M., Culbert. C.. Ambrose, R., Huber, E., Bluethmann, W. "Human-Robot Control Strategies for the NASADARPA Robonaut", Proceedings of the 2003 IEEE Aerospace Conference. Big Sky. Montana, March 8, 2003.

[6] Ambrose, R.O. and Savely. R.T.. "Synergy in the Design of Mobile Manipulation" SPIE 2001. Boston Mass, October 2001

[7] Ambrose R., Ambrose, C.. "Primate Anatomy, Kinematics. and Principles for Humanoid Design". International Journal of Humanoid Robotics, 2003. 